\title{
Accurate Calculation of Harmonics/Inter-Harmonics of Time Varying Load
}

\author{
A. K. Baliarsingh, I. Sihal, N. R. Samal, G. C. Martha, A. K. Mangaraj \\ Orissa Engineering College, Bhubaneswar, Odisha
}

\begin{abstract}
Between the harmonics of the power frequency voltage and current, further frequencies can be observed which are not integers of the fundamental. Totally eliminate the unacceptable spectra linkage error in the frequency domain caused by time domain signal. Using adaptive window with the numerical method is for exact calculation of harmonics/inter-harmonics components. This method adaptively adjusts the window width based on correlation calculation. The iterative algorithm does not require any knowledge without the system frequency. The only parameter needed is the signal sequence obtained by sampling the analog signal at equidistant sampling interval.
\end{abstract}

\section{INTRODUCTION}

The exact spectrum of a waveform provides a clear understanding of the causes and effects of waveform distortion. So in modern power system harmonics and interharmonics component are important for accurate analysis and measurement. In this case, the basic tool is discrete Fourier transform (DFT). DFT approximates the continuous Fourier transform of the time-domain signal. This approximation is a function of the waveform being analysed and the signal sequence covered by a window width. If the window width of DFT is not properly chosen there will be spectral leakage(1),(2).

In order to limit leakage effects, the classical Hanning window is used instead of the rectangular window. (3). Also windowing functions other than the rectangular window can reduce the spectral leakage to some extent. It is well known that the best solution of avoiding spectrum leakage is to select a window width that covers a period or an integer multiple of period of a signal. This is considered to be most challenging because the interharmonic frequencies are unpredictable and the system frequency may also vary.

Here show an adaptive algorithm to determine the window width through an iterative procedure based on correlation calculation. Given a signal sequence it is obtained by sampling a periodic time-domain waveform at equidistant interval. These eliminate the unwanted frequency leakage caused by truncation. Various case studies show that the proposed adaptive approach completely eliminates or significantly reduces the spectral leakage errors of DFT in the frequency domain.

In iterative algorithm there is not requiring any knowledge about the system frequency and the interharmonic constituents in the signal. The only parameter needed is the signal sequence obtained by sampling the analog signal at equidistant sampling interval. For long length signal period it finds the period for DFT such that the spectral lines by DFT exactly represent the continuous Fourier transform of the time-domain signal without any frequency leakage

\section{LITERATURE SURVEY}

There are some types of load for the generation of interharmonics. The generation of components in the sidebands of the supply voltage, frequency and its harmonics cause of changes in their magnitudes and/or phase angles. Due to rapid changes of current in equipment, or fluctuations in the voltage source as a result of interharmonices generate. Loads operating in a transient state continuously disturbances are generated. Largely these disturbances are random nature, depending on the load changes.

The other mechanism is the asynchronous switching (i.e. not synchronised with the power system frequency) of semiconductor devices in static converters are the main cause of interharmonices generation. Examples are pulse width modulation (PWM) converters and cycloconverters. Interharmonics generated by them may be located anywhere in the spectrum with respect to the power supply voltage harmonics. Interharmonics may be generated at any voltage level. These are transferred between these levels. Interharmonics generated in $\mathrm{HV}$ and MV systems and are injected into the LV system and vice versa. Their magnitude does not exceed $0.5 \%$ of the voltage fundamental harmonic. It may occur higher levels under resonance conditions.

Basic sources of interharmonices are:

1. Arcing loads

2. Electric motors

3. Double Conversion Systems

4. Cycloconverters

5. Time-Varying Loads 
6. Wind Turbines

7. Unexpected Sources

When transformers are subject to saturation and during switching processes interharmonics are generated.

\section{ADAPTIVE WINDOW WIDTH BY CORRELATION CALCULATION}

\subsubsection{Correlation Calculation of Discrete-Time Signals}

We obtain a discrete-time signal, when an analog signal is measured and recorded at equally spaced sampling interval, which is a vector having a sequence of values. The correlation of two discrete-time signals can be evaluated using inner product of two vectors.

In a vector space, an inner product operation for two finite length vectors and is mathematically defined as

$$
\langle f, g\rangle=\|f\|\|g\| \cos \theta
$$

Where the angle, $\theta \in|0, \Pi|$, is the angle between the two vectors in equation (1).

Where correlation coefficient $(k)$ is defined as $k \in|-1,1|$ based on (1) to quantify the degree of similarity between two signal sequences. If $\mathrm{k}=1$ is the maximum correlation when the two signals are parallel and in the same direction, and $\mathrm{k}=-1$ is the minimum correlation when the two signals are parallel and in the opposite direction

$$
k=\frac{\langle f, g\rangle}{\|f\|\|g\|}
$$

\subsubsection{Iterative Algorithm for Window Width Calculation}

The adaptive window width calculation algorithm is used .It searches for the most suitable window width in terms of local periodicity (or local similarity) of a signal sequence and complies with the periodicity requirement of DFT. When the correlation of two adjacent sections does not meet the defined termination criterion, the signal length of the first section is increased by one sample, and compared with the next adjacent section of the same length. The iteration continues until the termination criterion is satisfied.

Consider a discrete-time signal $\mathrm{S}$ of infinite size:

$$
S=\left\{s_{0} s_{1} s_{2} \ldots \ldots \ldots s_{p-1} s_{p} s_{p+1} \ldots \ldots . . s_{2 p-1} s_{2 p} s_{2 p+1} \ldots \ldots .\right\} .
$$

Step 1) let from $s_{0}$ to $s_{p-1}$ choosing for $\mathrm{p}$ samples from $\mathrm{S}$ signal as an initial value for iteration, and name it $X_{p}$.

Where $X_{p}=\left\{s_{0} s_{1} s_{2} \ldots \ldots \ldots s_{p-1}\right\}$

Step 2) Choose another samples from signal S, adjacent to $X_{p}$, and

Name it $Y_{p}$.

$$
Y_{p}=\left\{s_{p} s_{p+1} \ldots \ldots . . . s_{2 p-1}\right\}
$$

Step 3) Calculate the correlation between the two chosen sections $X_{p}$ and $Y_{p}$ using (2)

$$
K_{p}=\frac{\left\langle X_{p} Y_{p}\right\rangle}{\left\|X_{p}\right\|\left\|Y_{p}\right\|}
$$

$K_{p}$ is the periodicity indicator of p samples of signal sequence S.( Step 4) Test the periodicity.

If $K_{p}=1$ then $Y_{p}$ repeats $X_{p}$. Length of $\mathrm{p}$ samples is the period for the two sections. The spectral lines by DFT based on a window width of samples (or an integer multiple of samples) exactly represent the continuous Fourier transform of the time-domain signal without any frequency leakage. If $K_{p} \neq 1$, let $p=p+1$, and repeat Steps 1 to 4 until the specified termination criterion is satisfied.

\section{RESULTS AND DISCUSSION}

Interharmonics contain in ac/dc rectifier and a dc/ac inverter. The coupled reactor or capacitor in rectifier and inverter are called dc link. If the reactor or the capacitor has infinite value, there will be no ripples on the dc side. The ideal rectifier will only generate characteristic harmonics of 
$f_{h}=\left(p_{1} n \pm 1\right) f$

The dc side ripple frequency depends on the pulse number, the control method, and the inverter output frequency in CSI converters. The dc ripple contain frequencies expressed as follow

$f_{r}=n p_{2} f_{0}$

Where $p_{2}$ pulse number of the inverter

$f_{0}$ is the output frequency $\mathrm{n}$ is an integer number.

Here assume $f_{0}$ is set as $30, p_{2}=6, p_{1}=12$. If the inverter output frequency has a minor deviation from $30 \mathrm{~Hz}$, for example, $29.4 \mathrm{~Hz}$, the DC link reactor will has ripple frequency of $6 \times 29.6=176 \mathrm{~Hz}$. This ripple will beat with fundamental and consequently $50+176=236 \mathrm{~Hz}$ and $176-50=116 \mathrm{~Hz}$ interharmonics will be generated.

Fig 1 shows the Wave forms of AC-DC rectifier and DC-AC inverter the rectifier and inverter are coupled through a reactor or capacitor. The time domain of fundamental wave shape is shown in Fig. 2 and the frequency spectrum is shown in Fig. 3.

The window size used for spectrum analysis is 50 cycles, and sampling frequency is $3.2 \mathrm{kHz}$, which has resolution of the maximum common divider of all components, $1 \mathrm{~Hz}$. This size not ensure exactly integral cycles are not able to coverer in the window for all components hence leakage effect can not be eliminated. Fig. 4 Enlarged view of interharmonics at $302.8 \mathrm{~Hz}$, of AC-DC rectifier and DC-AC inverter the rectifier and inverter are coupled through a reactor or capacitor (using 50 cycle window width). It shows $303 \mathrm{~Hz}$.

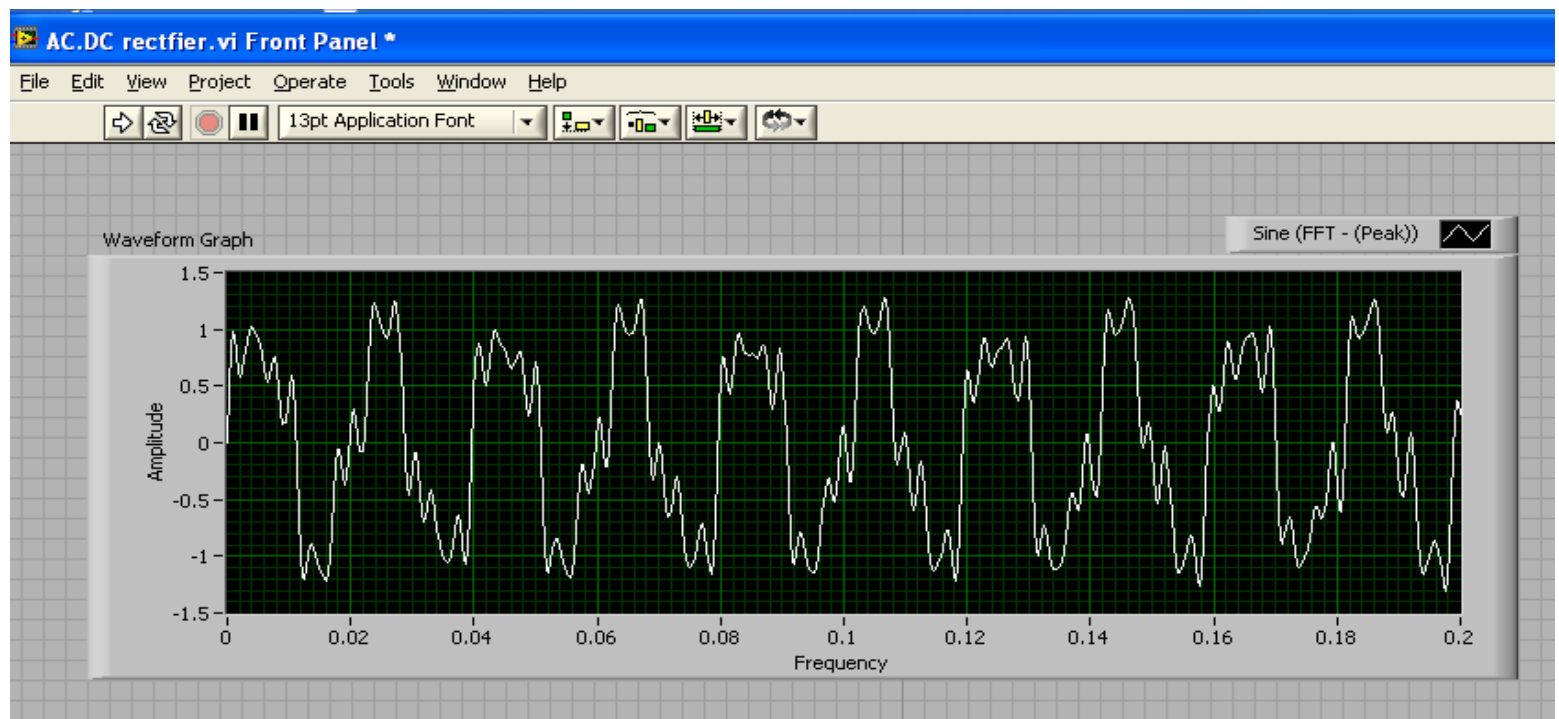

Fig. 1Wave forms of AC-DC rectifier and DC-AC inverter the rectifier and inverter are coupled through a reactor or capacitor.

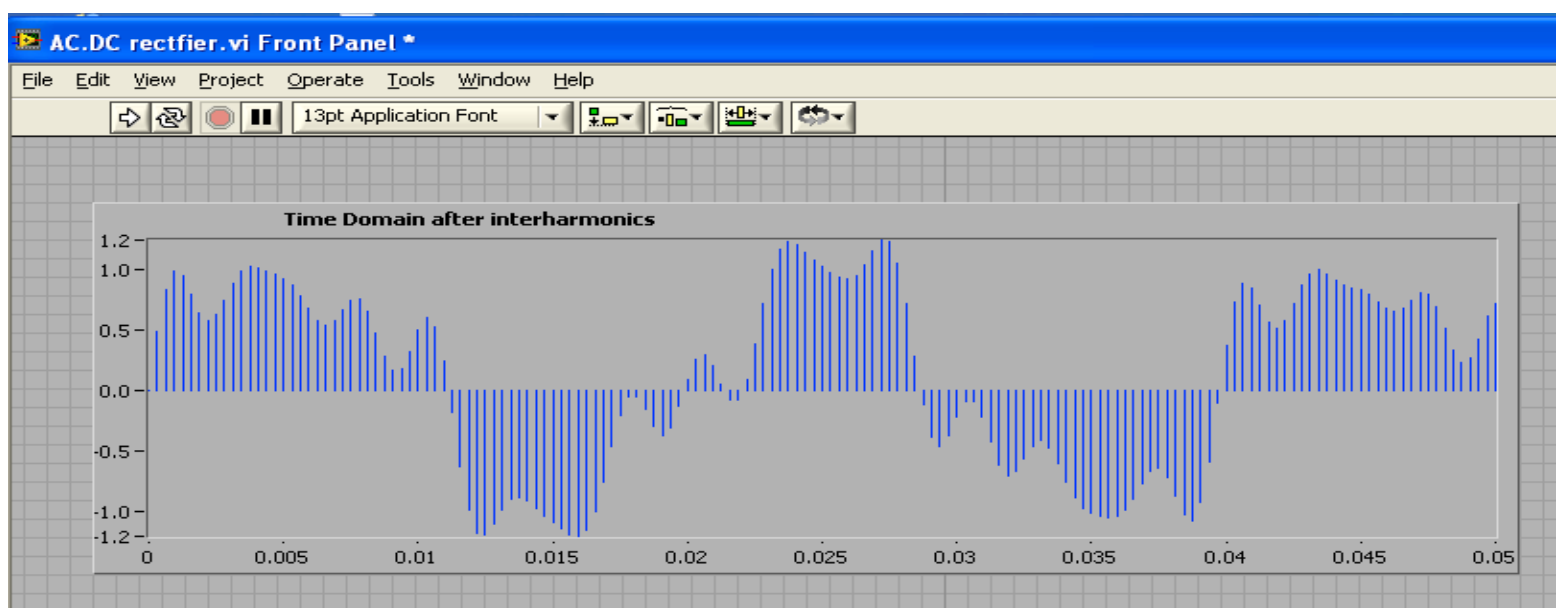

Fig. 2Time domain of AC-DC rectifier and DC-AC inverter the rectifier and inverter are coupled through a reactor or capacitor. 


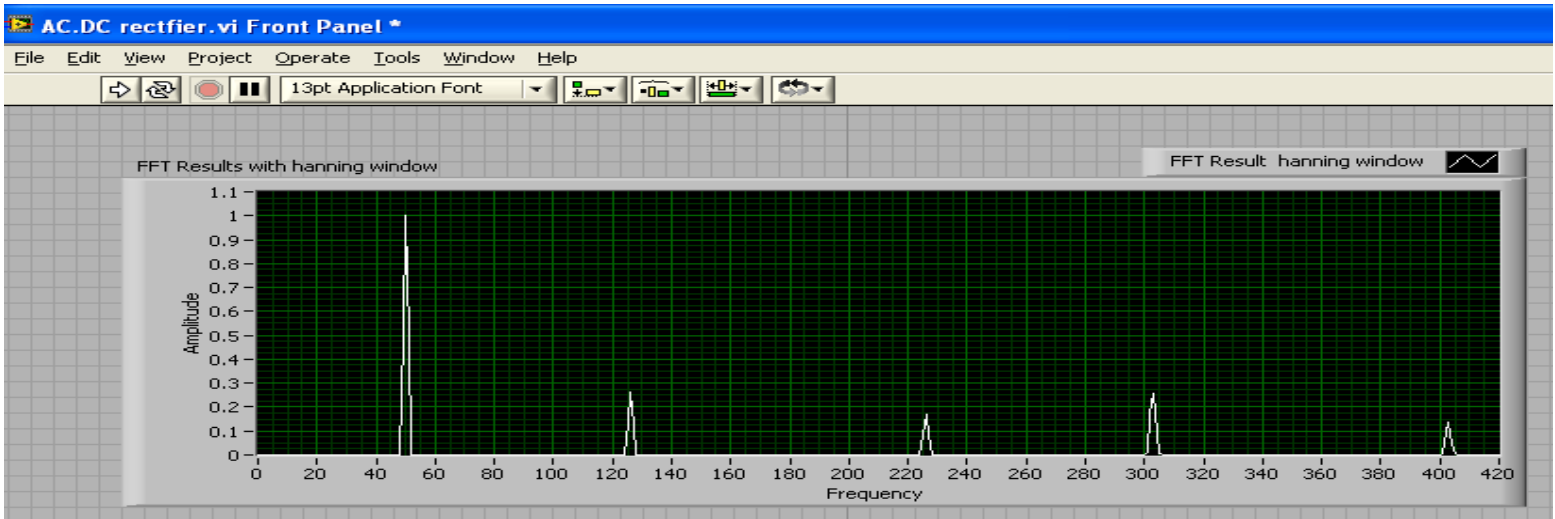

Fig. 3Magnitude spectrum of AC-DC rectifier and DC-AC inverter the rectifier and inverter are coupled through a reactor or capacitor (using 50 cycle window width).

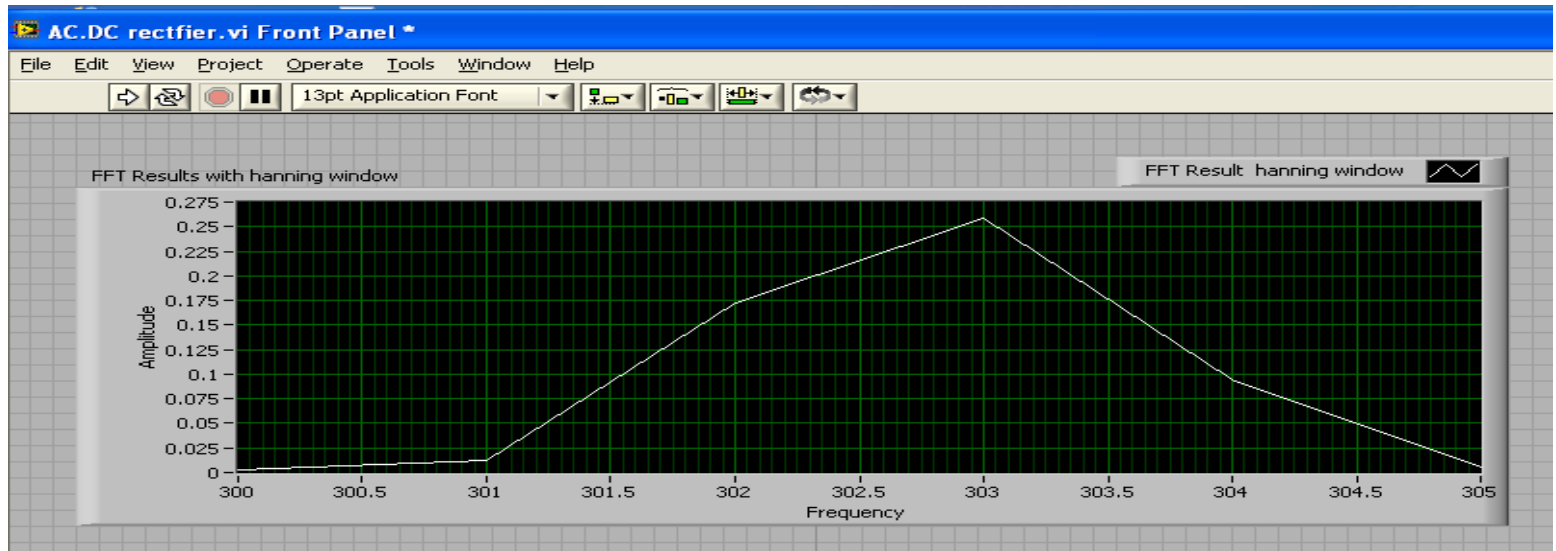

Fig. 4Enlarged view of interharmonics at $302.8 \mathrm{~Hz}$, of AC-DC rectifier and DC-AC inverter the rectifier and inverter are coupled through a reactor or capacitor (using 50 cycle window width).

\section{CONCLUSIONS}

Using adaptive window width the numerical method is for exact calculation of harmonics/interharmonics component. This method adaptively adjusts the window width based on correlation calculation. So eliminating the unwanted spectral leakage caused by truncation. The iterative algorithmdoes not require any knowledge about the system frequency and the interharmonic constituents. The only parameter needed is the signal sequence obtained by sampling the analog signal at equidistant sampling interval. Various case studies are done using chroma programming simulation data, show that the proposed algorithm provides an ideal numerical solution to the problem of spectral leakage encountered in power system harmonics and interharmonics analysis and detection.

\section{REFERENCES}

[1] R. C.Dugan , M. F. McGranagnan ,S. Santoso ,Electrical Power Systems Quality,New York:Mcgraw-Hill ,2003.

[2] Mattaveli P,Fellin L,Bordignon P,Perna M:Analysis of interharmonics in dc arc furnace installations, $8^{\text {th }}$ International Conference on Harmonics and Quality of Power,Athens, Greece,october14-16,1998.

[3] EN 50160:1999-Voltage characteristics of electricity supplied in public distribution systems.

[4] R. W. Ramirez, The FET. Fundamental and Concepts ,Englewood Cliffs ,Nj:Prentice-Hall,1985.

[5] E. Q. Brigham, The Fast Fourier Transform and its application, Englewood Cliffs, Nj. Prentice-Hall,1988.

[6] Interharmonic Task Force Working Document -IH0101 200001,IEEE.

[7] Arrillaga j, Watson N R,Chen S:Power system quqlity assessment, Wiley,2000.

[8] Kloss A:Oberschwingungen, Verlag vde, ISBN 3-8007-1541-4.

[9] Materials used in preparation of standard IEC 61000-2-4.

[10] Interharmonics in power system, IEEE interharmonic task force.

[11] J.Arrillaga, N. R. Watson, power system harmonics $2^{\text {nd }}$ New York,Willey,2004. 